\title{
Index rerum ad Vol. 160
}

\section{Confecit: C. Loeb-Schürch}

$(B)=$ Buchbesprechungen - Book reviews - Livres nouveaux $(V)=$ Vortrag - Report Communication

Abflussfazilität, v. Corticostéroides Ablatio, v. Carbon dioxide, Décollement rétinien Aderhaut, v. Melanoma, Natrium-

jodat, Zytomegalie Aide-lecture, v. Hemianopia Aimant à impulsion, v. Impuls-

magnetanlage Amblyopia, v. Congenital cataract Amblyopie strabique; nouveau

procédé de traitement de

Гamblyopie strabique à fixation

excentrique, 152 Amyloid, v. Dystrophia reticulata

corneae, Plasmozytom Anatomie, v. Orbital volume, X-ray

photos Angiomatosis; juxtapapillary retinal

angiomatosis. Histology of

congenital glaucoma. Transverse

ischaemic optic nerve necrosis in

neuroblastoma, 326 (V) Angiospastic maculopathy, v. Illumination tests Angle camérulaire, v.

Angiomatosis,

Limbus corneae Anomalies; symposium on surgical

and medical management of

congenital anomalies of the eye,

288 (B) Anophthalmos, v. Ectropion Apraxia; congenital ocular apraxia,

96 (V) Aqueous humour, v. Proteins Arteria ophthalmica, v. X-ray photos Arthropathy, v.

Cataracta

brunescens Atlas of strabismus, 288 (B) Atropinisation, v. Amblyopie

strabique

Augenkomplikationen, v. Cryo-surgery, Hyperpyrexie-Syndrom

Augenmuskeln, v. Apraxia, Stilling-Duane syndrome

Auto-photocoagulation in patients with Leber's disease, 121 (V)

Autoradiography, v. Vehicles

B $\beta$; atrophy of the optic nerve and vitamin B $\beta$ deficiency, 103 (V)

Biber-Haab-Dimmer disease,

v. Dystrophia reticulata corneae

Bindehaut, v. 35S

Biochemie, v. Natriumjodat

Blindness; the causes of blindness in 156 visually and mentally defective children, 292 (V)

Book reviews, 288, 430

Canalis opticus, v. X-ray photos

Carbon dioxide; the use of solid carbon dioxide in retinal detachment surgery, 112 (V)

Cat, v. Oxygen toxicity 
Cataract, v. Cataracta brunescens, Clinical demonstrations, Congenital Cataract, Cryo-surgery, Hyperpyrexie-Syndrom, Iris atrophy, Oxygen toxicity

Cataracta brunescens, deafness and arthropathy, 100 (V)

Cataracta congenita, v. Clinical demonstrations, Congenital cataract, Hyperpyrexie-Syndrom

Cécité, v. Blindness, Norrie's disease

Chamber angle, v. Angiomatosis, Limbus corneae

Champ magnétique, v. Impuls-magnetanlage

Index rerum

ad Vol. 160

433

Champ visuel, v. Fluorescein angio-graphy

Chat, v. Oxygen toxicity

Chirurgie, v. Carbon dioxide,

Congenital cataract, Cryo-surgery, Ectropion, Hyperpyrexie-Syndrom, Jones' operation, Ptosis

Chorioidea, v. Melanoma, Natrium-jodat, Zytomegalie

Chorioretinitis, v. Zytomegalie

Circulation, v. Fluorescein angio-

graphy

Clinical demonstrations, $105(\mathrm{~V})$

Coagulation solaire, v. Auto-photo-coagulation

Colour vision, v. Transmission

Complications oculaires, v. Cryo-surgery, Hyperpyrexie-Syndrom

Congenital cataract: surgical and visual problems. Two decades of development, 239

Congrès, v. Anomalies, Netherlands Ophthalmological Society

Conjunctiva, v. 35S

Contact lens; questions and answers on contact lens practice, 430 (B)

Cornea, v. Clinical demonstrations, Demonstrations, Dystrophia reti-culata corneae,

Hyalokerato-pathie, Limbus corneae

Corps étranger, v. Impulsmagnet-anlage

Corps vitré, v. Cryo-surgery, Hyalo-keratopathie, Palatoschisis

Corticostéroides; Faction des pro-duits anti-inflammatoires, corticostéroides et Tandéril, sur la tension intraoculaire, 403

Cowpox, v. Demonstrations

Cristallin, v. Clinical demonstrations, Congenital cataract, Demonstrations

Cryo-surgery; anterior vitreous changes following cataract extraction by cryo-surgery, 398

Cyst, v. 85S

Cytomégalie, v. Zytomegalie

Deafness, v. Cataracta brunescens,

Nome's disease Décollement rétinien, v. Carbon

dioxide

Décollement rétinien; anatomie pathologique et pathogénie du décollement rétinien, 25 (V)

Déficience mentale, $v$. Blindness, Norrie's disease

Dégénérescence maculaire, v. Clinical demonstrations

Dégénérescence tapéto-rétinienne, v. Dystrophia myotonica

Demonstrations; clinical demonstrations, 372 (V) 
Diabetes, v. Glaukom, Kimmelstiel-Wilson syndrome

Differential-Diagnostik, v. Clinical demonstrations, Melanoma, Plasmozytom, Stilling-Duane syndrome, Tumour pathology, Zytomegalie

Donders' Medal award; address to Professor Ashton on the occasion of the Donders' Medal award, $72(\mathrm{~V})$

Dysgenesis mesodermalis iridis, v. Limbus corneae

Dyslexia, v. Reading disorders

Dystrophia corneae verticillata, v. Clinical demonstrations, Demonstrations

Dystrophia myotonica; electro-retinographic and electro-encephalographic changes in dystrophia myotonica Steinert, 157

Dystrophia reticulata corneae; histopathology of the dystrophia reticulata corneae of Biber-Haab-

Dimmer, $321(\mathrm{~V})$

Echo-encephalography; proceedings in echo-encephalography, 431 (B)

Ectropion; operation for ectropion associated with anophthalmos, 379 (V)

EEG, v. Dystrophia myotonica, Transmission

Electrophysiology, v. Transmission

Electrophysiology; advances in electrophysiology and the visual system, 431 (B)

Elektroenzephalogramm, v. Dystrophia myotonica, Transmission

Elektronenmikroskopie, v. Leuco-dystrophy, Oxygen toxicity

434

Index rerum

ad Vol. 160

Elekt ookulogramm, v. Transmission

Elektrophorese, v. Décollement rétinien, Proteins

Elektroretinogramm, v. Dystrophia myotonica, Transmission

Emergencies; handbook of pediatric medical emergencies, 288 (B)

Endothelium, v. Hyalokeratopathie, Vehicles

Enucleation, v. Tumour pathology

Enzymhistochemie, v. Natriumjodat

EOG, v. Transmission

Epiphora, v. Jones' operation

Epithelium pigmentaire, v. Clinical demonstrations, Natriumjodat

Erblindungsursachen, v. Blindness, Norrie's disease

ERG, v. Dystrophia myotonica, Transmission

Exophorie, v. Heterophorietafel

Experimental research, v. Hyalokeratopathie, Natriumjodat, Oxygen toxicity, Vehicles

Exzentrische Fixation, v. Amblyopie strabique

Fabry's disease, v. Clinical demonstrations, Demonstrations

Facilité d'écoulement, v. Cortico-stéroides

Farbsinn, v. Transmission

Ferromagnetischer Fremdkörper, v. Impulsmagnetanlage

Fibroplasia retrolentalis, v. Blindness, Oxygen toxicity

Fixation excentrique, v. Amblyopie strabique

Fluo escein angiography of the optic disc in glaucoma, 331 (V)

Fonctions visuelles, v. Transmission 
Foramen opticum, v. X-ray photos

Foreign body, v. Impulsmagnetanlage

Fortbildung und Orientierung, 124, 202

Fremdkörper, v. Impulsmagnetanlage

Froboese's syndrome, v. Demonstrations

Frühgeburt, v. Blindness, Oxygen toxicity, Zytomegalie

Fundoskopie, v. Natriumjodat

Fundus flavimaculatus, v. Clinical demonstrations

Further studies and other information, 124, 202

Gefässe, v. Fluorescein angiography, Kimmelstiel-Wilson syndrome, Oxygen toxicity

Gefässneubildung, v. Oxygen toxicity

Genetics, v. Blindness, Cataracta brunescens, Demonstrations, Dystrophia reticulata corneae, Iris atrophy, Norrie's disease, Palato-schisis

Gesichtsfeld, v. Fluorescein angiography

Glaskörper, v. Cryo-surgery, Hyalokeratopathie, Palatoschisis

Glaucoma, v. Angiomatosis,

Fluorescein angiography, Mistake, Pemphigoid

Glaukom und Diabetes, 391

Gleichstrommagnet, v. Impulsmagnetanlage

Gonioskopie, v. Limbus corneae

Hemianopia; reading problems in

hemianopia, 322 (V) Hérédité, v. Blindness, Cataracta brunescens, Demonstrations, Dystrophia reticulata corneae, Iris atrophy, Norrie's disease, Palatoschisis Heterophorietafel; die

Heterophorietafel. Ein einfacher Test für die Untersuchung der Ruhestellung der Augen bei Beobachtungen eines Objektes im Leseabstand, 260 Hexosamin, v. Hyalokeratopathie

Histochemie, v. Natriumjodat Histologie, v. Angiomatosis, Hyalokeratopathie, Leucodystrophy, Limbus corneae, Melanoma, Oxygen toxicity, Plasmozytom, Zytomegalie Hornhaut, v. Clinical demonstrations, Demonstrations, Dystrophia reticulata corneae, Hyalokeratopathie, Limbus corneae Humeur aqueuse, v. Proteins Hyaloideo-retinal degeneration, v. Palatoschisis

Index rerum

ad Vol. 160

435

Hyalokeratopathie; die Histologie der experimentellen Hyalokeratopathie, 167

Hyalokeratopathie; experimentell erzeugte Hyalokeratopathie, 271 Hyaluronsäure, v.

Hyalokeratopathie Hypernephrom, v. Multiplizität Hyperoxie, v. Oxygen toxicity HyperpyrexieSyndrom; das Hyper-pyrexie-Syndrom als Kompli-kation nach intraokularen Ein-griffen am

Kleinkind, 385

Illumination tests; differential macular illumination tests, 409

Impressionstonometrie, v. Moses-effect

Imprint method, v. Orbital volume

Impulsmagnetanlage; ver gleichende experimentelle Feldstärke- und Kraftmessungen an einer neuen Impulsmagnetanlage und den konventionellen ophthalmologi-schen

Gleichstrommagneten, 195

Instrument, v. Carbon dioxide,

Hemianopia, Impulsmagnetanlage, Moses-effect, Videopupillography 
Intraokulardruck, v. Cortico-stéroïdes, Moses-effect

Iris, v. Limbus corneae, $35 \mathrm{~S}$

Iris atrophy; familial occurrence of iris atrophy and cataract, 291 (V)

Isotope, v. 3SS

Jodat-Retinopathie, v. Natriumjodat Jodazetat, v. Natriumjodat Jones' operation. A surgical

solution for difficult cases of epiphora,

$307(\mathrm{~V})$

Kammerwasser, v. Proteins

Kammerwinkel, v. Angiomatosis, Limbus corneae

Kaninchen, v. Hyalokeratopathie, Moses-effect, Natriumjodat, Orbital volume, Oxygen toxicity, Vehicles

Katarakt, v. Cataracta brunescens, Clinical demonstrations, Congenital cataract, Cryo-surgery, Hyperpyrexie-Syndrom, Iris atrophy, Oxygen toxicity

Katze, v. Oxygen toxicity

Kimmelstiel-Wilson syndrome; the favorable influence of Nandro-lone-Decanoate on the eye and kidney in some cases of Kimmel-stiel-Wilson syndrome. Some problems in assessing kidney biopsies, $378(\mathrm{~V})$

Kongresse, v. Anomalies, Netherlands Ophthalmological Society

Lähmung, v. Stilling-Duane syndrome

Lapin, v. Hyalokeratopathie, Moses-effect, Natriumjodat, Orbital volume, Oxygen toxicity, Vehicles

Leber's disease, v. Auto-photo-coagulation

Lens, v. Clinical demonstrations, Congenital cataract, Demonstrations

Lesehilfe, v. Hamianopia

Leucodystrophy; metachromatic leucodystrophy, 2 (V)

Leukokorie, v. Norrie's disease, Ophthalmopathologie, Retino-blastom

Lider, v. Ptosis

Limbus corneae; the deep structure of the limbus corneae, 85 (V)

Linse, v. Clinical demonstrations, Congenital cataract, Demonstrations

Lipidose, v. Leucodystrophy

Livres nouveaux, 288, 430

Macula, v. Auto-photocoagulation,

Illumination Maculadegeneration, v. Clinical

demonstrations Magnetfeld, v. Impulsmagnetanlage Maus, v. Oxygen toxicity Melanin, v.

Natriumjodat Melanoma; malignant melanoma of

the choroid. An unusual variant,

314 (V) Melanoma, v. Mistake, Tumour

pathology Melanozytoblastom, v. Multiplizität Membrana hyaloidea persistens,

v. Clinical demonstrations

436

Index rerunt ad Vol. 160

Mental deficiency, v. Blindness,

Norrie's disease Methode, v. Amblyopie strabique,

Carbon dioxide, Congenital

cataract, Ectropion, Heterophorie- 
tafel, Illumination tests, Orbital

volume, Schielverdächtige, Video-

pupillography Microscopie électronique, v. Leuco-

dystrophy, Oxygen toxicity Mistake; an unnecessary mistake,

369 (V) Moses-effect; significance of the

Moses-effect and the 'central area'

of the footplate with regard to the

findings of tonometry, 129 Motilität, v. Apraxia, Stilling-Duane

syndrome Mouse, v. Oxygen toxicity Mucopolysaccharide, v. Cataracta

brunescens, Hyalokeratopathie Multiplizität; Beitrag zur primären

Multiplizität maligner Tumoren,

227 Muscles oculaires, v. Apraxia,

Stilling-Duane syndrome Myelom, v. Plasmozytom Myopia; on the etiology of myopia,

$431(\mathrm{~B})$

Nahsehen, v. Heterophorietafel

Nandrolone-Decanoate, v. Kimmel-stiel-Wilson syndrome

Natriumjodat; zur Histochemie der Natriumjodat- und Natriumjod-azetat-Retinopathie, 176

Near vision, v. Heterophorietafel

Nerf optique, v. Angiomatosis, Auto-photocoagulation, Bß, Leuco-dystrophy, X-ray photos

Netzhaut, v. Clinical demonstrations, Décollement rétinien, Demonstrations, Dystrophia

myotonica, Illumination tests, Leucodystro-phy, Natriumjodat, Oxygen toxicity, Palatoschisis,

Reflections of light, Transmission, Zytomegalie

Netzhautgefässe; Klinik und Patho-logie der Netzhautgefässe, 430 (B)

Netherlands Ophthalmological Society, Lustrum Congress 75th Anniversary, 159th Meeting

(1967), 1-123 (V)

Netherlands Ophthalmological Society, 160th Meeting (1967), 291-384 (V)

Neuroblastoma, v. Angiomatosis

Neuroophthalmologie, v. Leuco-dystrophy, Transmission

Norrie's disease, $375(\mathrm{~V})$

Oa, v. Oxygen toxicity Operation, v. Carbon dioxide,

Congenital cataract, Cryo-surgery, Ectropion, Hyperpyrexie-Syndrom, Jones' operation, Ptosis

Ophthalmic artery, v. X-ray photos Ophthalmopathologie; die Ophthal-

mopathologie. 1.Teil, 124 Ophthalmopathologie; die Ophthalmopathologie. 2. Teil, 202 Optic

disc, v. Fluorescein angio-

graphy Optic foramen, v. X-ray photos Opticus, v. Angiomatosis, Auto-photocoagulation, B $\beta$, Leuco-dystrophy, X-ray photos Orbital volume; the imprint method to determine orbital volume in the rabbit, 142 Outflow facility, v. Corticostéroïdes Oxygen toxicity; some aspects of the comparative pathology of oxygen toxicity in the retina, $54(\mathrm{~V})$

Paedoophthalmologie, v. Blindness, Hyperpyrexie-Syndrom, Oxygen toxicity, Schielverdächtige Palatoschisis; hereditary hyaloideo-retinal degeneration and palatoschisis, 384 (V)

Palsy, v. Stilling-Duane syndrome

Papille, v. Fluorescein angiography

Papillitis, v. Auto-photocoagulation

Parese, v. Stilling-Duane syndrome

Paupières, v. Ptosis

Pemphigoid; three patients from the pemphigoid group and a case of pigment glaucoma, 120 (V) 
Perfectionnement et orientation, 124, 202

Persistent primary vitreous, v. Clinical demonstrations

Phorometer, v. Heterophorietafel

Index rerum

ad Vol. 160

437

Physiologie des voies et des centres visuels, 430 (B)

Pigmentepithel, v. Clinical demonstrations, Natriumjodat

Pigmentglaukom, v. Pemphigoid

Plasmozytom; zur Klinik und Pathologie des Plasmozytoms am Auge, 217

Prematurity, v. Blindness, Oxygen toxicity, Zytomegalie

Pression oculaire, v. Cortico-stéroïdes, Moses-effect

Prisme redresseur, v. Amblyopie strabique

Proceedings, v. Anomalies, Netherlands Ophthalmological Society

Proliferation vasculaire, v. Oxygen toxicity

Protein, v. Décollement rétinien

Proteins; the proteins of the pathologic human aqueous humour. An in vivo investigation, 280

Prothese, v. Ectropion

Pseudoglioma, v. Nome's disease

Ptosis; correction of ptosis. A surgical-anatomical approach, 306 (V)

Pupillography, v. Videopupillo-graphy

Rabbit, v. Hyalokeratopathie, Moses-effect, Natriumjodat, Orbital volume, Oxygen toxicity,

Vehicles Radiologie, v. X-ray photos Radiotherapie, v. Plasmozytom,

Retinoblastom Reading disorders, 319 (V) Reading helps, v. Hemianopia Recherches

expérimentales, v. Hyalokeratopathie, Natriumjodat, Oxygen toxicity, Vehicles Reflections of

light from the ocular

fundus, 303 (V) Retina, v. Clinical demonstrations, Décollement rétinien, Demonstrations, Dystrophia myotonica, Illumination tests, Leuco-dystrophy, Natriumjodat, Oxygen toxicity, Palatoschisis, Reflections of light, Transmission, Zytomegalie Retinal detachment, v. Carbon dioxide, Décollement rétinien

Retinitis centralis serosa, v. Illumination tests

Retinoblastom; über Behandlungs-ergebnisse beim Retinoblastom (1960-1968), 231

Retinopathia diabetica, v. Glaukom, Kimmelstiel-Wilson syndrome

Retrolental fibroplasia, v. Blindness, Oxygen toxicity

Rieger's dysgenesis, v. Limbus comeae

$35 \mathrm{~S}$; injection of radioactive $35 \mathrm{~S}$. A new technique in the treatment of iris and conjunctival cysts, $308(\mathrm{~V})$

Sauerstoff, v. Oxygen toxicity

Schielen, v. Amblyopie strabique, Schielverdächtige

Schielverdächtige; zur Methodik der objektiven Untersuchung schielverdächtiger Kleinkinder, 421

Schiøtz'sches Tonometer, v. Moses-effect

Schwachsinn, v. Blindness, Norrie's disease

Schwalbe'scher Ring, v. Limbus corneae

Sehzellen, v. Natriumjodat 
Sodium iodate, v. Natriumjodat

Souris, v. Oxygen toxicity

Squint, v. Amblyopie strabique, Schielverdächtige

Steinert'sche myotonische Dystro-phie, v. Dystrophia myotonica

Stilling-Duane syndrome; the differential diagnosis between the Stilling-Duane syndrome and lateral rectus palsy, $98(\mathrm{~V})$

Strabismus, v. Amblyopie strabique, Schielverdächtige

Strahlentherapie, v. Plasmozytom, Retinoblastom

Subluxatio lentis, v. Demonstrations

Sun-coagulation, v. Auto-photo-coagulation

Surdité, v. Cataracta brunescens, Norrie's disease

Surgery, v. Carbon dioxide, Congenital cataract, Cryo-surgery, Ectropion, Hyperpyrexie-

Syndrom, Jones' operation, Ptosis

438

Index rerum ad Vol. 160

Syndrome, v. Clinical demonstrations, Demonstrations, Hyper-pyrexie-Syndrom, Leucodystrophy, Kimmelstiel-Wilson-Syndrom, Stilling-Duane syndrome

Synophthalmus, v. Demonstrations

Tandéril, v. Corticostéroïdes Tapetoretinale Degeneration,

v. Dystrophia myotonica Tapetum lucidum, v. Demonstrations Taubheit, v. Cataracta

brunescens,

Norrie's disease Tension oculaire, v. Corticostéroïdes,

Moses-effect Therapie, v. Amblyopie strabique, Congenital cataract, Jones' operation,

Plasmozytom, Ptosis, Retinoblastom, 35S Tonometry, v. Moses-effect Toxocara, v.

Demonstrations Toxoplasmose, v. Zytomegalie Trabeculum corneo-sclerale,

v. Limbus corneae Transmission; symposium: the transmission of information by the retina under normal and pathological conditions, 75 (V) Tritium thymidine, v. Vehicles Tryptophan loading test, v. B6 Tumor, v. Angiomatosis, Melanoma, Mistake, Multiplizität, Plasmozytom, Retinoblastom, Tumour pathology Tumour pathology; intraocular tumour pathology and its relation to clinical ophthalmology, $18(\mathrm{~V})$

Vaisseaux, v. Fluorescein angio-graphy, Kimmelstiel-Wilson syndrome, Oxygen toxicity

Vehicles; ophthalmic vehicles. II. Comparison of ointment and polyvinyl alcohol 1.4\%, 265

Vererbung, v. Blindness, Cataracta brunescens, Demonstrations, Dystrophia reticulata corneae, Iris atrophy, Norrie's disease, Palatoschisis

Verhandlungsberichte, v. Anomalie: Netherlands Ophthalmological Society

Vessels, v. Fluorescein angiographj Kimmelstiel-Wilson syndrome, Oxygen toxicity

Videopupillography; a new video-pupillography, 248

Virus, v. Zytomegalie

Vision de près, v. Heterophorietafel

Visual cells, v. Natriumjodat

Visual field, v. Fluorescein angio-graphy

Visual function, $v$. Transmission

Vitamin, v. B $\beta$

Vitelliforme Degeneration, v. Demonstrations

Vitreous body, v. Cryo-surgery, Hyalokeratopathy, Palatoschisis 
Waardenburg lecture, v. Limbus corneae X-ray photos of the optic foramen, 354 (V) Zirkulation, v. Fluorescein angio-

g. graphy Zyste

v. 85S Zytomegalie; Augenveränderungen

bei Zytomegalie

209 